\title{
Using Smartphone in Health-Care
}

\author{
Abdulrahman Almudhi \\ College of Engineering, \\ University of Bridgeport, 06604, \\ Connecticut, United States
}

\begin{abstract}
This paper aims to review the effects of smartphones in the medical field and investigate some of their usages in the healthcare area that can be very helpful in the future.

\section{Methodology \\ Conducting a comprehensive literature review on smartphone- based system formed the theoretical foundation of this paper.}

\section{Findings}

The model shows that medical related apps, smartphone uses in medical devices, smartphone based health parameters monitoring, and medical survey using smart phones are four example of approaching smartphones to the medical device.
\end{abstract}

\section{Research limitations/implications}

The research that was used is limited to around 30 papers from relevant conferences within the particular industry of interest.

\section{Originality/value}

This paper shows the approaching of smartphones to the hospitals and health field generally, and how that is important.

\section{Keywords}

Smartphone-based using in health-care

\section{INTRODUCTION}

Nowadays, there is growing in smartphones usage in medical field. Smartphone has been involved and used in clinical application, such as, colorimetric imaging [1-5]. The important reason for that is the skyrocketing that happened in nanotechnology, which allow the specialist to integrate biosensors and bioelectronics with these smartphones. In spite of this fast improving in this technology, we still in the first step in this failed [6]. Therefore, researches that improving biosensors and bioelectronics in smartphone-based need to be completed and supported [7]. Hidalgo-Mazzei, Reinares [8] reported that being this troublesome, cell phones are likewise the most PCs as such. They are conveyed all around, and used to run all kind of capacities, the vast majority of which are personal to the clients. With regards to human services, the pattern of looking for wellbeing data from the web is a conspicuous alternative on versatile stages, yet the genuine change got through the surge of applications composed by designers to serve a wide assortment of medicinal and social insurance situations, for example, wellbeing training, intercession and adherence upgrade, and in addition prescription and analysis. Applications focused on wellbeing experts, patients, and general society, as medicinal references, number crunchers, through the best approach to being connections or contrasting options to therapeutic gadgets [8]. Generally, what doctors and patients needed to access on stationary PCs have been conveyed to them by applications right onto their hands/pockets, enlarged by creative utilization of the new detecting capacities that required beforehand unique gear, outside to the figuring gadget [9].

\section{RESEARSH METHOD}

Recently smartphone-based methods e.g. [4] p. 273 became increasingly important in particular when the goal is the health-care "Smartphone-based portable biosensing system [5] p. 81." One form for "Smart phones: new clinical tools in oncology" [10] p. 429 is the review centric research approach in which a researcher reviews "The use of smartphone applications by urology trainees" [6] p. 263, but the argument can be that also our knowledge still do not have enough technologies. In the research offered here the focus is on joining the most important "Using smartphone in health field " [2]. In this study I recognize the most vital cell phones uses in the medicinal field that help with the present business or progressive situation to fabricate our goal to accomplish a high ground. My investigation approach melds the "Prosperity parameters seeing by mobile phone for individual fulfillment change" in which a rich depiction of each consider our present affiliation setting is developed [11] p. 615 .

\section{SMARTPHONE USAGE IN MEDICINE}

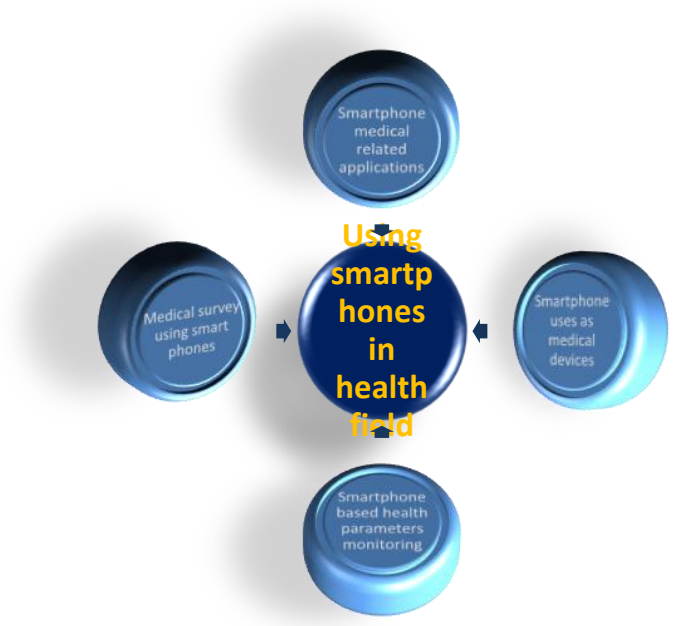

Fig 1: Different smartphone usages in medical field.

\subsection{Using smartphone in health field}

Recently, smartphone has been involved and used in clinical application, such as, colorimetric imaging [3]. The important reason for that is the skyrocketing that happened in nanotechnology, which allow the specialist to integrate biosensors and bioelectronics with these smartphones. In spite of this fast improving in this technology, we still in the first step in this failed. Therefore, researches that improving biosensors and bioelectronics in smartphone-based need to be completed and supported [7]. 


\subsubsection{This Optical biosensors using in smartphone-based}

Please use a 9-point Times Roman font, or other Roman font with serifs, as close as possible in appearance to Times Roman in which these guidelines have been set. The goal is to have a 9-point text, as you see here. Please use sans-serif or non-proportional fonts only for special purposes, such as distinguishing source code text. If Times Roman is not available, try the font named Computer Modern Roman. On a Macintosh, use the font named Times. Right margins should be justified, not ragged.

\subsubsection{Smartphone-based microscopic bio imaging} Imaging in medical field frameworks on cell phone were are in view of a few imaging endeavors utilizing highlight telephones to picture cells and microbes with magnifying instruments. Using a camera-empowered mobile for microscopy imaging in hematologic and irresistible illnesses [12]. The infinitesimal gadget and imaging results are appeared in Fig. 1a. The morphologies of platelets and microorganisms can be imaged with the PDA based magnifying instrument in splendid field and fluorescence mode, separately [13, 14]. At that point, a few research bunches have embedded PDA into magnifying instrument gadgets for various biomedical applications [15].

\subsubsection{Smartphone-based fluorescence biosensors} delivered with the littler amplifying instrument synchronously to satisfy fluorescence named IDs for DNA, contamination, and nanoparticles [4]. At in the first place, in effective procedure used fluorescence to stamp tests to progress the assurance of imaging. Fluorescent amplifying instrument on cell phone frequently had advanced assurance than that of impressive field amplifying instrument in microscopy imaging. In any case, to catch and measure Nano scale objects, focus should be made by correspondence out of both extra point of convergence and camera point of convergence of mobile phone. It much of the time required far reaching volume of optical association and extended process flightiness in imaging. In order to overcome this issue, Zhang, Jiang [4] planned to assess fluorescent power in place of fluorescent consider the banner label for analyze area with wireless.

\subsubsection{SPR biosensors using in smartphone}

Optical sensors were ordinarily suggested as surface plasmon resonation (SPR) sensors. These sensors used refract metric recognizing to size excitation of surface plasmons. In the last years, SPR sensors had boundless improvements with respect to development change and their functions [4]. For finds out about biosensing with wireless, SPR estimations were in like manner finished by coupling optical refract metric creation to mobile phone on the commence of photosensitive sensor arrange [4]. electrochemical biosensors using in smartphone

Electrochemistry has shown wide employments of quantitatie area for fundamental examinations, such as, proteins, metabolites, nucleic acids in security checking, nature appraisal, and scientific diagnostic, inferable from its unflinching quality, port-limit, ease, and simplicity [15].

\subsection{Smartphone medical related applications}

Smartphones applications have started getting into medical device strongly and quickly. That is why there are a lot of studies that try to find out the secrets of this technology and try to do the best efforts to get more benefits [16]. For example, smartphones goniometry apps help doctors to measure joint angle. Moreover, Interne connection availably has contributed to increase smartphones with medical field worker. Contentiously, many of them have medical apps that help to keep them up to date with latest information, which they need in their every day work [10]. On the other hand, we are still in the first level of medical smartphones applications that will open a wide door to improve medical field and medical devices [9]. A few applications are particularly imperative to radiologists. Some radiologists regard the change of restorative applications for convenient stages [10]. A couple of utilizations hope to keep oncologists and distinctive clinicians aware of everything with respect to information that is significantly vital to their common work. A couple of applications have been made for iPhone and iPad, each application containing cases and articles relate to body area (midriff, musculoskeletal, stomach, neurology). These cases can be very helpful and guided wherever and at whatever point.

The iRadiology App, this application contains a point by point posting of numerous radiology incidents that engage practicing radiologists, and remedial understudies to improve their affirmation aptitudes of radio graphs, CT, and MRI pictures. iPhone zooming can be handy when it comes to focusing on diverse parts of photo. Versatile MIM, furthermore fuses pictures from many medical devices such as ultrasound. Blend pictures from cross breed CT scanners are covered and combined to give most outrageous structural feature [17].

mobilePDR: An application gives a cross platform service that is open on the most working systems and smart pads structures. mobilePDR overhauls a full summary of pharmaceuticals which are starting now supported by Food and Drug Adminestration [17]. NCCN or The National Comprehensive Cancer Network Clinical Practice Guidelines in Oncology are in like manner obtainable through a flexible application made for smart phones [17].

Various applications are moreover profitable to clinicians who require the newest investigation headways obtainable anywhere they are. An online development journal in app store which called Ecancer; later off erings built-in video from ASCO 2010. What's more, furthermore flexible applications for smoking suspension, there are a couple on chest care, involving iBreastCheck, which consolidates recordings to demonstrate to females' best practices to check their individual chests, reports on month to month chest checks, and information on the most capable strategy to upgrade your lifestyle to reduce malady possibility [17].

\subsection{Smartphone uses as medical devices}

With the technology innovation, the smartphone will be a very good substitution for many medical devices [18]. However, this is will happen only after a lot of researches and studies to be sure of the sufficient and safety these devices [19]. In addition, these mobile phones has an inside and out sketched out affiliation structures which can trade data viably without the necessities for pay a large number of dollars for wired affiliation [20]. In addition, sensors can be fused in phones adequately, which is to an awesome degree profitable in various tests [21, 22]. Finally, the development pushed will be continue with quickly and we will have the ability to find phone based progressions with a sensible cost in the nearest future [23]. Likewise, the present advancement in wireless based affirmation propels our interests in the change of new uncommonly tried and true and speedy illustrative devices in 


\section{FA acknowledgment [24].}

Electrocardiogram (ECG) has been using for more than hundred years for the checkup of ischemic heart illness and cardiovascular arrhythmias [25]. New development at present considers a regulated one lead recording is going to be found by a modernized checking bipolar contraption which is joined [25]. The average bipolar member leads of the regular twelve leads ECG can be obviously imitated with unpretentious bipolar relationship of the members which is known as Einthoven's triangle. Three leads (VR, VL, VF) can in like manner be rehashed obviously by interfacing the looking at cathode to the thought extremity and the negative post which connect the other two limbs to of the recording bipolar contraption. Likewise, (V1, V2, V3, V4, V5, V6) leads can be made with a bipolar positioning: the positive lead is on the waist and the negative conductor is on the lower left leg. In one experiment, there was an attempt to duplicate the 12 lead ECG using the bipolar arrangement to cut edge cell advancement [25]

One study utilized the hear device screen combined with an cell phone and ECG application. Indeed, even though the study discovered extremely goal-oriented result, this cell phone therapeutic gadget needs a great deal to discover its way to this present reality [25]

\subsection{Smartphone based health parameters monitoring}

Every day, there are hundreds of new applications for smartphones in many fields. Some of them are apps medical application, which help either the doctor, technician, and the patient [11]. Smartphone has proved its powerful to work as a real-time monitor [26]. The advantage of that is the tiny place of smartphones, easy data transection, and low price of sensors and smartphone integration, even though there are some exceptions [27]. There are many examples for using smartphones as a monitor medical device, such as, Electrocardiogram ECG, despite the fact that these critical devices needs more time to ensure their safety and validity $[28,29]$.

The relentlessly extending change in helpful phone use has made offered novel human-machine edges, for instance, touchscreens, high and sensible data charges of flexible frameworks, and new convincing and speedy essentialness capable chip [30]. Furthermore, varying sensors not positively associated with correspondence are joined, for instance, some semiconductors ,such as, Complementary Metal-Oxide Semiconductor (CMOS) contraptions of the cameras to shot images, and GPS for road pilot or to discover likely loss of the phone, accelerometers to distinguish the turn of the monitor. Each one of these sensors allow going the wireless from a contact machine to a device for watching prosperity parameters and highlight of life [31].

Owed a broad diffusing comprehensive, around 93\% of world masses are phone handlers. Regardless, there are in like way shared sicknesses spread over the world, that can be confined or better cured through enduring prosperity seeing by phones [32]. Europe has the most extraordinary middle age in the planet, where nearby $20 \%$ of the people is old 65 and senior. Mobile phone is an inestimable financing for guaranteeing a shielded space and wonderfulness of life change by watching disorders identified with the musculoskeletal system and drop acknowledgment for the more settled.

\subsubsection{Smartphone kinematic sensor}

The kinematic sensor qualities make it appropriate for estimations on the individual body. The fundamental believed is that the body position is connected with that of the mobile phone [32]. For instance, the examination of the animating data identified by the kinematic sensor licenses singular drop acknowledgment, or horrendous body positions are obvious by interfacing the PDA screen region to the manager's neck point of view [33].

\subsubsection{Phone's camera}

The phone's camera has many features that able it to be used as Blood Pressure monitoring system, Heart Rate extracting system, blood $\mathrm{SpO} 2$ concentration measuring system, and also it is used for the eyes examination [32, 34].

\subsubsection{Phone's microphone}

The phone's speaker has unbelievable affectability and repeat response. These traits allow finding of pathologies by considering the breath and hack sound. PDAs in like manner has adjunctive beneficiary to take after racket and dismissal it. This extends the way of the made banner and appropriately the consistency of the checking. These components help for hack examination, and racket related signs affirmation [32].

\subsection{Medical survey using smart phones}

Nowadays, smartphone is with everybody and sometimes you can find it with even the very young people. Therefore, it is very useful as a tool to complete surveys in many different fields; one of them is the medical. There are many surveys related to medical devices has been done using mobile phones, for example, given out to urology trainees in Ireland to evaluate their ownership [6]. One advantage cannot be found in other survey, which is getting the result as soon as user finished the survey [35]. In the nearest future, surveys for medical field will happen only using these smart devices in order to get make the process of a survey very easy, quick and useful [36].

Utilizing of cell phones as a part of social insurance is drawing closer as nothing unexpected. People have constantly utilized possible capacities to enhance their most essential activities and protect their most significant properties; and not any more important than their private wellbeing. The utilization of data and correspondence innovation in the practice of human services presented the possibility of eHealth, where interchanges is permitting telemedicine, PCs are taking care of wellbeing information, and the Internet is putting forth the base to trade a wide range of wellbeing data and assistances [37, 38]. At the point when adaptability got to be likely, media communications happened through cell phones, and CPU moved alongside people as light tablets and after that convenient gadgets. The eHealth pushed to incorporate voyaging wellbeing (mHealth); yet at the same time, the wireless was just a telephone and the $\mathrm{PC}$ was just a PC; till both joined into a solitary component called a "cell phone" [9, 39].

In one survey, More than $90 \%$ of the used sample has a smartphone, with $80 \%$ of those willing to use their personal device inside the office. With around $50 \%$ of smartphone handlers having medical apps and above $85 \%$ having the Internet to approach medical information, this would propose that online resources give a lot to medical activities. With numerous trusts that this present practice, it can be valued to further grow software that recognizes the possible of mobile admission to information that is authorized [35]. 


\section{DISCUSS MODEL}

A smartphone is described as a phone enabled for message or Internet use [6, 40, 41]. Applications are downloadable programs that meet a definite job. Smartphone usage in the hospital offers direct access to specialist medical and healthcare information, causing extra effective decision making, this in effect decreases medical mistakes and developed communication [22, 42]. Moreover, mobiles use purposes as an operational enabler of public commitment. There is an argue that there are tow mechanisms behind this fact: information and communication, and social connectivity have been allowed[36, 43].

\section{RESULT AND DISSCUTION}

Starting late, various adaptable sensor gadgets have been upgraded for grouped applications, for instance, $\mathrm{pH}$ distinguishing, cell checking and food section examination [44]. The smaller sensor devices joined with distinguishing circuits were delivered into irrelevant and humbler size, that help to make complex interest to get advancement of limit and show adjust. Mobile phones, was a respectable choice to control estimation and show comes about as a result of its phenomenal limits [4]. The mobile phone based system was used to perceive all through ongoing impedance checking using the peptide changed cathodes [5].

\section{CONTRIBUTION AND NEW INSIGHT}

Biosensors and bioelectronics on phone were produced from bio-recognizing attempts working out of date clear cellphones, just called highlight phones. Different studies have masterminded biosensor and bioelectronics traces using mobiles for helpful social protection diagnostics external well-reserve workrooms [45, 46]. Notwithstanding the way that colossal improvement has been done, there are difficulties in frameworks. Employments of mobile phones based biosensors and bioelectronics. For optical sensor structures, optical parts and electronic cameras as pictures traded the signs. After that, the photo unobtrusive components can be bankrupt around phone or sent to the servers through remote frameworks. In this manner, much imperativeness should be taken to diminish optical associations for phone. Novel imaging frameworks ought to be used to redesign advancements of optical associations. For example, minute associations can be dynamically arranged trapped on negligible contraptions using novel picture strategies with a specific end goal to lessening masses of the structures [47].

\section{CONCLUSION}

This review has presented the summary of smartphone for bio-detections and another usage. The smartphones-based sensors, until now, can be ordered into optical biosensors, SPR biosensors, electrochemical biosensors, and NFC biosensors on smartphone. With the improvement in smartphones technology, it can provide an excellent mobile platform, such as cell imaging, and environmental monitoring. Moreover, the smartphone will provide low-cost, portable and efficient alternative medical devices or a handy tool that help doctors, nurses, and specialists who work in hospitals. The new news scope has focused on a creating stress in making apps for the restorative field factors checking to growth the individual fulfillment. This concern is supported by the constant dissemination of the phone in the most countries in the world, like the example of illnesses necessitating the steady seeing of prosperity considerations. Moreover, the purpose of this effort is to give a close diagram on the precision and trustworthiness of every phone based restorative administrations application concerning standard helpful contraptions and techniques. To engage the legitimate prudent dialog, the likely use of the sensors planning is moreover presented. To support the understand ability of the paper the applications are shown on the preface of the sensors that they send. The inadequacy and qualities of the applications are emphasized with a particular true objective to make a fundamental examination of why it could be a considerable.

\section{REFERENCES}

[1] Su, K., et al., High-sensitive and high-efficient biochemical analysis method using a bionic electronic eye in combination with a smartphone-based colorimetric reader system. Sensors and Actuators B: Chemical, 2015. 216: p. 134-140.

[2] Vashist, S.K., et al., Graphene-based rapid and highlysensitive immunoassay for C-reactive protein using a smartphone-based colorimetric reader. Biosensors and Bioelectronics, 2015. 66: p. 169-176.

[3] Vashist, S.K., et al., A smartphone-based colorimetric reader for bioanalytical applications using the screenbased bottom illumination provided by gadgets. Biosensors and Bioelectronics, 2015. 67: p. 248-255.

[4] Zhang, D., et al., Smartphone-based portable biosensing system using impedance measurement with printed electrodes for 2,4,6-trinitrotoluene (TNT) detection. Biosensors and Bioelectronics, 2015. 70: p. 81-88.

[5] Zhang, D. and Q. Liu, Biosensors and bioelectronics on smartphone for portable biochemical detection. Biosensors and Bioelectronics, 2016. 75: p. 273-284.

[6] Nason, G.J., et al., The use of smartphone applications by urology trainees. The Surgeon, 2015. 13(5): p. 263 266.

[7] Mei, Q., et al., Smartphone based visual and quantitative assays on upconversional paper sensor. Biosensors and Bioelectronics, 2016. 75: p. 427-432.

[8] Hidalgo-Mazzei, D., et al., Signs and Symptoms Selfmonitoring and Psychoeducation in Bipolar Patients with a Smart-phone Application (SIMPLe) Project. European Psychiatry, 2015. 30, Supplement 1: p. 320.

[9] Hussain, M., et al., The Landscape of Research on Smartphone Medical Apps: Coherent Taxonomy, Motivations, Open Challenges and Recommendations. Computer Methods and Programs in Biomedicine, 2015.

[10] Senior, K., Smart phones: new clinical tools in oncology? The Lancet Oncology, 2011. 12(5): p. 429 430 .

[11] Lamonaca, F., et al., Health parameters monitoring by smartphone for quality of life improvement. Measurement, 2015. 73: p. 82-94.

[12] Abbas, Z., et al., 2013 Index IEEE Sensors Journal Vol. 13. Ieee sensors journal, 2013. 13(12): p. 5005.

[13] Choi, S., et al., Medical applications of atomic force microscopy and Raman spectroscopy. Journal of nanoscience and nanotechnology, 2014. 14(1): p. 71-97.

[14] Shen, L., J.A. Hagen, and I. Papautsky, Point-of-care colorimetric detection with a smartphone. Lab on a Chip, 2012. 12(21): p. 4240-4243.

[15] Zhang, D. and Q. Liu, Biosensors and bioelectronics on 
smartphone for portable biochemical detection. Biosens Bioelectron, 2016. 75: p. 273-84.

[16] Hidalgo-Mazzei, D., et al., Signs and Symptoms Selfmonitoring and Psychoeducation in Bipolar Patients with a Smart-phone Application (SIMPLe) Project. European Psychiatry. 30, Supplement 1: p. 320.

[17] Zangheri, M., et al., A simple and compact smartphone accessory for quantitative chemiluminescence-based lateral flow immunoassay for salivary cortisol detection. Biosens Bioelectron, 2015. 64: p. 63-8.

[18] Hirschorn, D.S., et al., Use of Mobile Devices for Medical Imaging. Journal of the American College of Radiology, 2014. 11(12, Part B): p. 1277-1285.

[19] Baquero, G.A., et al., Surface 12 lead electrocardiogram recordings using smart phone technology. Journal of Electrocardiology, 2015. 48(1): p. 1-7.

[20] Georgiadis, P., et al., Remote monitoring of electromagnetic signals and seismic events using smart mobile devices. Computers \& Geosciences, 2009. 35(6): p. 1296-1303.

[21] Zangheri, M., et al., A simple and compact smartphone accessory for quantitative chemiluminescence-based lateral flow immunoassay for salivary cortisol detection. Biosensors and Bioelectronics, 2015. 64: p. 63-68.

[22] Zhang, D., et al., Protein Detecting with Smartphonecontrolled Electrochemical Impedance Spectroscopy for Point-of-care Applications. Sensors and Actuators B: Chemical.

[23] Walzik, M.P., et al., A portable low-cost long-term livecell imaging platform for biomedical research and education. Biosensors and Bioelectronics, 2015. 64: p. 639-649.

[24] Yang, X., et al., A portable system for on-site quantification of formaldehyde in air based on Gquadruplex halves coupled with A smartphone reader. Biosens Bioelectron, 2015. 75: p. 48-54.

[25] Baquero, G.A., et al., Surface 12 lead electrocardiogram recordings using smart phone technology. J Electrocardiol, 2015. 48(1): p. 1-7.

[26] Yang, X., et al., A portable system for on-site quantification of formaldehyde in air based on G- quadruplex halves coupled with A smartphone reader. Biosensors and Bioelectronics, 2016. 75: p. 48-54.

[27] Jovašević-Stojanović, M., et al., On the use of small and cheaper sensors and devices for indicative citizen-based monitoring of respirable particulate matter. Environmental Pollution, 2015. 206: p. 696-704

[28] El Menshawy, M., A. Benharref, and M. Serhani, An automatic mobile-health based approach for EEG epileptic seizures detection. Expert Systems with Applications, 2015. 42(20): p. 7157-7174.

[29] Lou, D., et al., A Wireless Health Monitoring System based on Android Operating System. IERI Procedia, 2013. 4: p. 208-215.

[30] Carolan, E., S.C. McLoone, and R. Farrell, Comparing and contrasting smartphone and non-smartphone usage. 2013.

[31] Daponte, P., et al., State of the art and future developments of measurement applications on smartphones. Measurement, 2013. 46(9): p. 3291-3307.

[32] Lamonaca, F., et al., Health parameters monitoring by smartphone for quality of life improvement. Measurement, 2015.

[33] He, Y., Y. Li, and S.-O. Bao. Fall Detection by built-in tri-accelerometer of smartphone. in Biomedical and Health Informatics (BHI), 2012 IEEE-EMBS International Conference on. 2012. IEEE.

[34] Kwon, S., H. Kim, and K.S. Park. Validation of heart rate extraction using video imaging on a built-in camera system of a smartphone. in Engineering in Medicine and Biology Society (EMBC), 2012 Annual International Conference of the IEEE. 2012. IEEE.

[35] Patel, R.K., et al., A UK perspective on smartphone use amongst doctors within the surgical profession. Annals of Medicine and Surgery, 2015. 4(2): p. 107-112.

[36] Ingrams, A., Mobile phones, smartphones, and the transformation of civic behavior through mobile information and connectivity. Government Information Quarterly, 2015.

[37] Hovenga, E.J., et al., Health informatics-an introduction. Studies in health technology and informatics, 2009. 151: p. 9-15. 